\title{
Higher order pair corrections to electron-positron annihilation
}

\author{
A.B. Arbuzov*
}

Dipartimento di Fisica Teorica, Università di Torino;

INFN, Sezione di Torino, via Giuria 1, I-10125 Torino, Italy

\begin{abstract}
Radiative corrections due to pair production in process of electron-positron annihilation are considered. The main attention is paid to the corrections of the third order. Simultaneous emission of photons and pairs is taken into account. The leading log approximation and convolution procedure are used. Exponentiation is discussed. Theoretical uncertainty in description of higher order secondary pairs is estimated.
\end{abstract}

PACS: 12.20.--m Quantum electrodynamics, 12.20.Ds Specific calculations

\section{Introduction}

The high statistics and accuracy of experimental measurements at LEP and SLC result in the outstanding level of precision in definition of the Standard Model parameters (see Ref. [1, 2] and references therein). In this situation effects of radiative corrections are of great importance.

One of particular contributions to the process of electron-positron annihilation is the radiation of secondary pairs. In comparison with the photon radiation, it is relatively small, because it appears only starting from the $\mathcal{O}\left(\alpha^{2}\right)$ order. Nevertheless, the total effect of pair production can reach several permil (or even e few percent in particular cases) and should be taken into account. We will consider here the reactions of the following type:

$$
e^{+}+e^{-} \rightarrow f+\bar{f}+\left(f^{\prime} \bar{f}^{\prime}(\gamma)\right)
$$

where $f \bar{f}$ is the so-called primary fermionic pair, $f^{\prime} \bar{f}^{\prime}$ is the secondary one, and $(\gamma)$ stands for the usual photon radiation, which can take place in our case starting from the third order $\mathcal{O}\left(\alpha^{2}\right)$. The primary pair is the one registered in the particular measurement. The secondary one can be either real or virtual, and has to be accounted as a radiative correction according to certain experimental conditions (with some restrictions (cuts), if required). In principle, the pairs can be of the same flavour, like in the process $e^{+} e^{-} \rightarrow 2 \mu^{+} 2 \mu^{-}$. When both pairs are seen in the detector, one has to apply an algorithm to choose the primary pair.

\footnotetext{
*The present address: Bogoliubov Laboratory of Theoretical Physics, JINR, Dubna, Russia.
} 
The complete second order QED calculation for production of the initial state secondary pairs $\left(e^{+} e^{-}, \mu^{+} \mu^{-}\right.$, and hadrons) was performed in Refs. 3, 4. The corresponding final state effect was considered in Ref. [5].

The interference between amplitudes for the initial and final state pair production has not been calculated systematically. An estimate of this effect will be presented in the Conclusions.

It is observed, that the $\mathcal{O}\left(\alpha^{2}\right)$ approximation is not adequate to provide the desirable precision, especially in the region close to the $Z$-peak. From another side, at LEP2, when the radiative return is allowed, QED radiative corrections reach several hundred percent and should be evaluated with care. In order to achieve the precision, required by the experiments (see Ref. [2]), one should consider higher orders of the perturbation theory. Another important point is that in the $Z$-peak region the sub-leading corrections are comparable in size with the leading ones. So, one has to go beyond the leading log approximation (LLA).

The goal of the present paper is to describe and discuss the treatment of pair corrections, implemented in the semi-analytical code ZFITTER v.6.30 [6]. The main attention will be paid to the third order corrections. Some numerical illustrations will be given. The uncertainty of the approach will be analyzed in the Conclusions.

\section{Pair corrections in higher orders}

Starting from the $\mathcal{O}\left(\alpha^{3}\right)$ order, one has amplitudes with simultaneous emission of pairs and photons. We treat them as a part of pair corrections, because they have not been taken into account in the traditional photonic RC. The main subject of this article is to analyze this effect.

As usually in $e^{+} e^{-}$annihilation, the most important contributions are from the initial state (IS) radiation (real and virtual). In a typical high-energy experiment, the conditions of the Kinoshita-Lee-Nauenberg theorem are fulfilled for the final state (FS) radiation. That is why the large logarithms (mass singularities) cancel out in the FS correction, and the latter becomes relatively small. From the other hand, a specific energy dependence of the kernel cross section can provoke an enhancement of the IS correction, like it happens at the $Z$-peak or in the radiative return to the resonance at LEP2.

Pair corrections to the process of $e^{+} e^{-} \rightarrow f \bar{f}$ become ambiguous, when one observes 4fermion final states. Nevertheless, the bulk of the pair RC to the 2-fermion observables is coming from the kinematical situations, when the secondary pair is either virtual or escapes registration (in the beam pipe or because of a small energy). On could try to subtract from the experimental data all real secondary pairs by means of a Monte Carlo generator. But that would destroy the cancellation between the real and virtual pairs, and the corrections would become even more pronounced and dangerous. One can get a serious numerical instability, trying to subtract both the virtual and the real pairs by means of different codes. Even if the subtraction of the pure second order pairs is evaluated perfectly, we have to keep in mind the higher order corrections due to instantaneous emission of pairs and photons. The cancellation between the virtual and real pair $\mathrm{RC}$ is important not only in the $\mathcal{O}\left(\alpha^{2}\right)$, but in higher orders as well. Indeed, we would end up with a completely wrong third order corrections (the error would exceed the entire effect), if we were using for the subtraction of virtual and real pair contributions two different codes, one with the third order pair-photon effect and another one without it. 

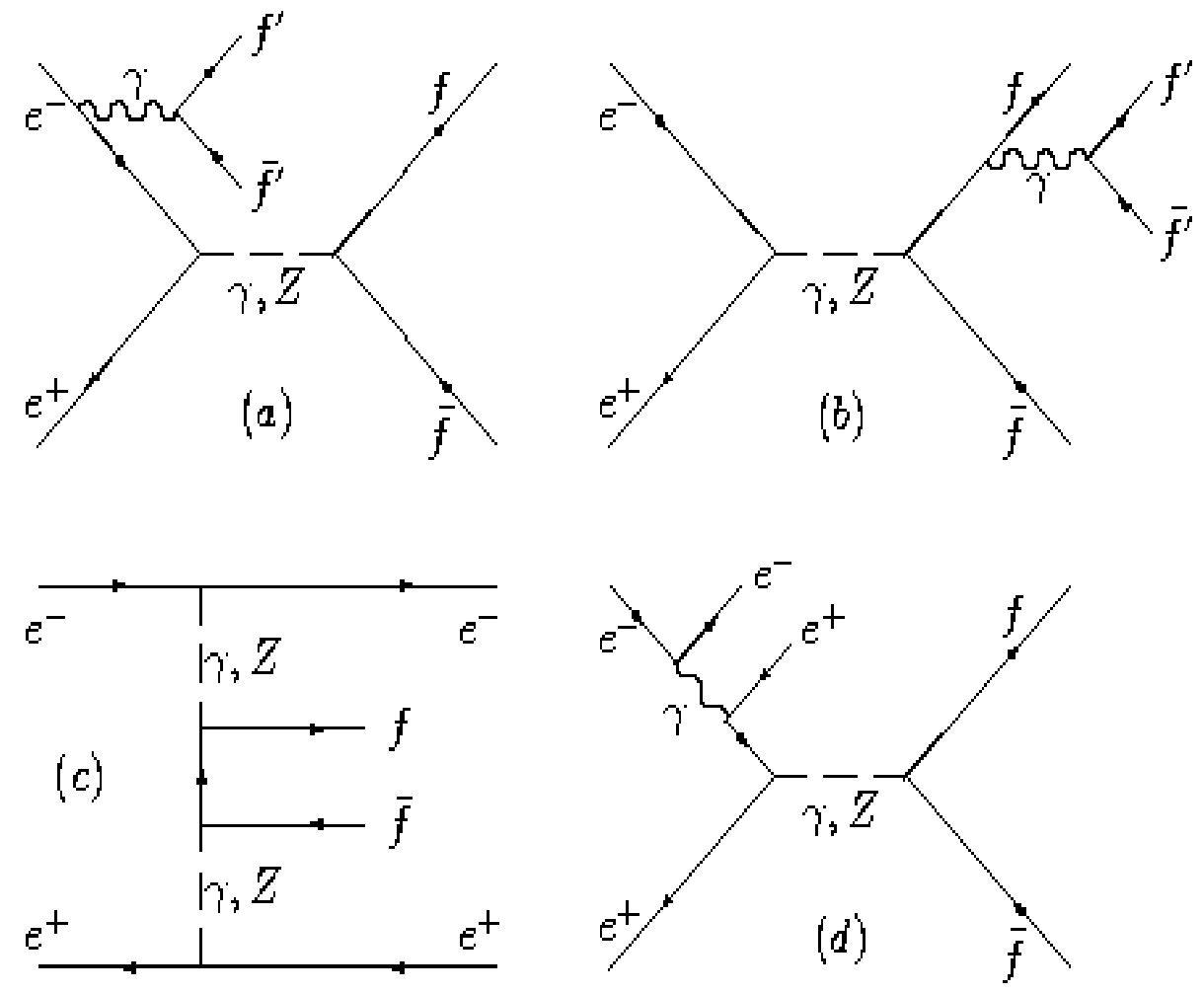

Figure 1: Different types of Feynman diagrams for real pair production.

Here we will follow the definition of signal and background according to Feynman diagrams, as discussed in Ref. 2]. In Figure 1 we show several representatives of Feynman graphs for real pair production processes. Only the diagrams Fig. $1 a$ and Fig. $1 b$ (plus the ones with pair production via a virtual photon emitted from the initial state positron of the final state antifermion $\bar{f}$ ) are to be accounted as the signal. These diagrams describe the non-singlet (NS) mechanism of pair production. The diagrams of the type $(a)$ and $(b)$ with the production of a secondary pair via a virtual $Z$-boson are treated as background. The so-called multi-peripheral process is described by Fig. 1c. Diagrams of type $(d)$ represent the singlet mechanism of pair production. Their contribution is considered as a background too. The pure electroweak fourfermion processes with $W$-boson intermediate states fall into a separate class [2] from both the theoretical and the experimental points of view. The relevant (signal) Feynman graphs for virtual pair corrections are given in Figure 2.

In other words we will count only the non-singlet secondary pairs produced via virtual photons Fig. 1 $a, b$ (some numbers for the singlet pairs will be presented ). The contribution from the so-called background diagrams, like multi-peripheral, double-resonant $(Z Z)$, and singlet pair production, are supposed to be subtracted from the data. Interferences between the signal and background amplitudes are typically small, but some of them, like the ones between the IS singlet and non-singlet pairs, should be taken into account. We will not use any direct cut on the phase space volume of real secondary pairs (an inclusive treatment of radiative events). Only the condition (see Eq. (3i)) on the primary pair invariant mass $s^{\prime}$ will be applied (the dependence on the secondary pair invariant mass will be shown for the FS correction). 


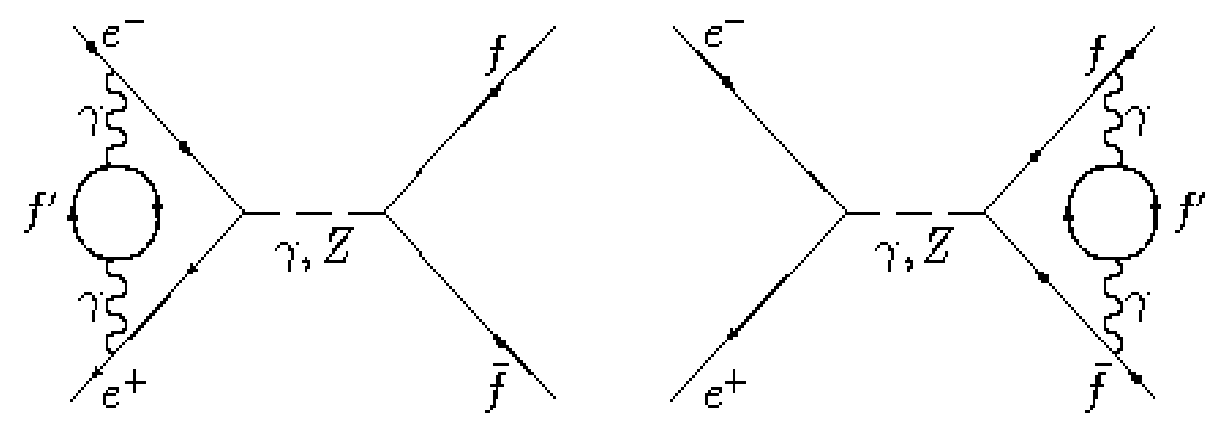

Figure 2: Feynman diagrams for virtual pair corrections.

\subsection{The final state pair $\mathrm{RC}$}

In the lowest order for the FS pair corrections we use the results of Ref. [5]. Our aim here is to estimate the effect of higher orders. Concerning the interplay with the IS photonic corrections, it is claimed, that the final state pair corrections should be realized in the multiplicative way:

$$
\sigma=\sigma_{\mathrm{Born}}\left(1+\delta_{\mathrm{IS} \gamma}\right)\left(1+\delta_{\mathrm{FSP}}\right)
$$

where $\delta_{\mathrm{IS} \gamma}$ stands for the initial state photonic correction, and $\delta_{\mathrm{FSP}}$ gives the final state pair one. At LEP2 energies, when the radiative return to the $Z$-peak is allowed, we have huge values of $\delta_{\mathrm{IS} \gamma}$ (typically above 100 percent), and the additive summation of the corrections $\left(1+\delta_{\mathrm{IS} \gamma}+\delta_{\mathrm{FSP}}\right)$ fails completely. In principle, the initial state pair correction should participate in formula (2) together with the photonic one, but, as one can see from numerical illustrations, an additive treatment of the IS and FS pair corrections is acceptable as well.

The effect of simultaneous photon and pair radiation from the final state can be estimated by multiplying the corresponding contributions: $\mathcal{O}(\alpha)$ FS photon RC and the $\mathcal{O}\left(\alpha^{2}\right)$ FS pair $\mathrm{RC}$. One can see that this source does not give more than 0.05 permil in the accepted signal definition. This estimate was confirmed also by a computation (analogous to Eq. (5) ) of the $\mathcal{O}\left(\alpha^{3}\right)$ LLA result for the FS non-singlet pairs. It is worth to note here, that additional strong cuts on the final state photons and pairs can increase the FS corrections and revive the problem of the corresponding missing higher order terms.

In Table 1 we give the value of correction $\delta_{\mathrm{FSP}}$ due to the final state pair production in the process of annihilation into muons as a function on cuts on the invariant mass of the primary and secondary pairs;

$$
\begin{aligned}
M_{\text {primary }}^{2} & \equiv s^{\prime} \geq z_{\min } s, \quad M_{\text {secondary }}^{2} \leq s P_{\text {cut }}, \\
\delta_{\mathrm{FSP}} & =10^{3}\left(\frac{\sigma_{\mathrm{ISP}, \mathrm{FSP}, \gamma}^{\mathrm{RC}}}{\sigma_{\mathrm{ISP}, \gamma}^{\mathrm{RC}}}-1\right)
\end{aligned}
$$

where $\sigma_{\mathrm{ISP}, \gamma}^{\mathrm{RC}}$ is the cross section with the photonic and IS pair corrections taken into account. One can see that the dependence on the invariant mass of the secondary pair is very weak. That confirms our expectations, because the contribution of large-invariant-mass pairs $\left(M_{\text {secondary }}^{2} \gg m_{f}^{2}\right.$, where $m_{f}$ is the mass of a pair constituent; $m_{f}=m_{\pi}$ for the case of hadronic pairs) does not contain any large logarithm, and so it is suppressed by the relative factor $1 / L^{2}$. The same should be valid for the IS pairs: the relative contribution of large-invariant-mass 


\begin{tabular}{|c|c|c|c|c|c|c|}
\hline$E_{\mathrm{cm}}[\mathrm{GeV}] \backslash P_{\mathrm{cut}}$ & 0.0001 & 0.001 & 0.01 & 0.1 & 0.3 & 0.99 \\
\hline & \multicolumn{7}{|c|}{$z_{\min }=0.01$} \\
\hline 88.1867 & -0.47 & -0.13 & +0.11 & +0.18 & +0.19 & +0.20 \\
91.1867 & -0.48 & -0.13 & +0.11 & +0.19 & +0.20 & +0.20 \\
94.1867 & -0.48 & -0.13 & +0.11 & +0.19 & +0.20 & +0.20 \\
189 & -0.57 & -0.12 & +0.14 & +0.22 & +0.23 & +0.23 \\
206 & -0.59 & -0.11 & +0.14 & +0.22 & +0.23 & +0.23 \\
\hline & \multicolumn{7}{|c|}{$z_{\min }=0.1$} \\
\hline 88.1867 & -0.51 & -0.18 & +0.05 & +0.12 & +0.12 & +0.12 \\
91.1867 & -0.51 & -0.18 & +0.05 & +0.12 & +0.12 & +0.12 \\
94.1867 & -0.52 & -0.18 & +0.05 & +0.12 & +0.12 & +0.12 \\
189 & -0.62 & -0.18 & +0.06 & +0.13 & +0.14 & +0.14 \\
206 & -0.64 & -0.18 & +0.06 & +0.13 & +0.14 & +0.14 \\
\hline 88.1867 & -1.08 & -0.89 & -0.82 & -0.81 & -0.81 & -0.81 \\
91.1867 & -1.09 & -0.91 & -0.83 & -0.83 & -0.83 & -0.83 \\
94.1867 & -1.10 & -0.91 & -0.84 & -0.83 & -0.83 & -0.83 \\
189 & -1.38 & -1.14 & -1.05 & -1.05 & -1.05 & -1.05 \\
206 & -1.42 & -1.17 & -1.08 & -1.08 & -1.08 & -1.08 \\
\hline 88.1867 & -1.68 & -1.62 & -1.61 & -1.61 & -1.61 & -1.61 \\
91.1867 & -1.70 & -1.63 & -1.63 & -1.63 & -1.63 & -1.63 \\
94.1867 & -1.70 & -1.64 & -1.63 & -1.63 & -1.63 & -1.63 \\
189 & -2.13 & -2.04 & -2.04 & -2.04 & -2.04 & -2.04 \\
206 & -2.19 & -2.11 & -2.10 & -2.10 & -2.10 & -2.10 \\
\hline
\end{tabular}

Table 1: Radiative correction $\delta_{\mathrm{FSP}}^{e^{+} e^{-} \rightarrow \mu^{+} \mu^{-}}$in permil for different cuts.

secondary pairs is negligible. This statement concerns, of course, pair production via virtual photons, while the double-resonant $Z Z$-mechanism (or $W W$ ) has to be treated separately.

\subsection{The initial state pair $\mathrm{RC}$}

The combination of the initial state pair correction with the final state photonic one should be treated in the same way as Eq (2), while it is not so important numerically. We will concentrate now on the main problem, when both the pair and the photons are produced in the initial state.

The initial state pair contributions to the corrected cross section is presented as the convolution of the boosted Born cross section with the so-called pair radiator:

$$
\delta \sigma^{\text {pair }}=\int_{z_{\min }}^{1} \mathrm{~d} z \tilde{\sigma}(z s) H(z)=\sigma(s) H_{\Delta}+\int_{z_{\min }}^{1-\Delta} \mathrm{d} z \tilde{\sigma}(z s) H_{\Theta}(z)
$$

Where $H_{\Delta}$ represents the impact of virtual and soft (small-energy) pairs, $\Delta$ is an auxiliary parameter $\left(m_{e}^{2} / s \ll \Delta \ll 1\right)$; numerical results should be independent of its value. In higher orders, when we have instantaneous emission of pairs and photons, we will still call such 
corrections as the pair ones. In our computations the $\mathcal{O}\left(\alpha^{2}\right)$ formula for the pair radiator is taken from Refs. 3. 4 .

Going beyond the lowest order, one starts usually with the leading logarithmic approximation. Indeed, the main contribution to the radiative correction under consideration is coming from the electron pair production, because it is reinforced by powers of the large logarithm $L_{e} \equiv \ln \left(s / m_{e}^{2}\right)$ (the corresponding leading logs for pairs of other flavours can be constructed as well). There are two different mechanisms for electron-positron pair creation in our process: the singlet and non-singlet ones. The first one is absent for non-electron pairs.

At the $Z$-peak it is worth to try to re-sum higher orders, because the convergence of the perturbation theory there is slow. The first exponentiated formula for the IS LLA pair production was suggested in Ref. [7. The process of one pair production was supplied there by emission of an arbitrary number of photons. This approach gives a reasonable approximation for the leading corrections close to the $Z$-peak. But it does not include some significant nextto-leading terms, and even the known third order leading logs are not reproduced completely.

Another way [8] is to use the Mellin transformation to solve the QED evolution equation for soft and collinear photonic contributions supplied by the running QED coupling constant. The authors received a simultaneous exponentiation of photonic and pair corrections, which takes into account important next-to-leading terms. Unfortunately, their formulae require additional evaluations (inverse Mellin transformations). Moreover, the applicability of their method to the mixed pair-photon corrections is not clear.

In Ref. 9] a phenomenological formula for simultaneous exponentiation of photonic and pair radiation was proposed. The correspondence of the exponentiated formula to the perturbative results was shown for the case of real hard radiation. Nevertheless, the structure of the radiator function, suggested in [9], does not allow to verify analytically the correspondence for the soft and virtual part of the corrections.

\subsubsection{The leading logarithmic approximation}

Let us start our evaluations with the leading logarithmic approximation. It will serve us as a guide. The third order LLA radiator function can be taken from the Dokshitzer-GribovLipatov-Altarelli-Parisi evolution equation (we dropped below the pure photonic corrections):

$$
\delta \sigma_{e}^{(3) \text { pair }}=\int \mathrm{d} z \tilde{\sigma}(z s)\left(\frac{\alpha}{\pi}\left(L_{e}-1\right)\right)^{3}\left[\frac{5}{24} P^{(1)} \otimes R^{s}(z)-\frac{1}{36} R^{s}(z)+\frac{1}{6} P^{(2)}(z)+\frac{1}{27} P^{(1)}(z)\right]
$$

where

$$
R^{s}(z)=\frac{1-z}{3 z}\left(4+7 z+4 z^{2}\right)+2(1+z) \ln z
$$

is the singlet splitting function;

$$
\begin{aligned}
P^{(1)}(z) & =\lim _{\Delta \rightarrow 0}\left\{\delta(1-z)\left(2 \ln \Delta+\frac{3}{2}\right)+\Theta(1-\Delta-z) \frac{1+z^{2}}{1-z}\right\} \\
P^{(2)}(z) & =\lim _{\Delta \rightarrow 0}\left\{\delta(1-z)\left[\left(2 \ln \Delta+\frac{3}{2}\right)^{2}-\frac{2 \pi^{2}}{3}\right]\right. \\
& \left.+\Theta(1-\Delta-z) 2\left[\frac{1+z^{2}}{1-z}\left(2 \ln (1-z)-\ln z+\frac{3}{2}\right)+\frac{1+z}{2} \ln z-1+z\right]\right\}
\end{aligned}
$$


are the non-singlet splitting functions. The convolution of the singlet splitting function $R^{s}$ with the first order non-singlet one is known [10]:

$$
\begin{aligned}
P^{(1)} \otimes R^{s}(z) & =\left(\frac{3}{2}+2 \ln (1-z)\right) R^{s}(z)+(1+z)\left(-\ln ^{2} z+4 \operatorname{Li}_{2}(1-z)\right) \\
& +\frac{1}{3}\left(-9-3 z+8 z^{2}\right) \ln z+\frac{2}{3}\left(-\frac{3}{z}-8+8 z+3 z^{2}\right) .
\end{aligned}
$$

\subsubsection{Beyond the LLA}

But, as we learned from the $\mathcal{O}\left(\alpha^{2}\right)$ level from the complete calculations, the contribution of the sub-leading correction in the region close to the $Z$-peak is very large (say, about one half of the leading one). In order to account the most significant part of the sub-leading corrections we consider the convolution of the $\mathcal{O}\left(\alpha^{2}\right)$ pair radiator with the ordinary $\mathcal{O}(\alpha)$ photonic radiator, proportional to the $P^{(1)}$ splitting function. In this way we receive the main part of the $\mathcal{O}\left(\alpha^{3}\right)$ leading logs, proportional to $P^{(2)}$, and the sub-leading terms enhanced by $\ln (1-z) /(1-z)$, like $L^{2} \ln (1-z) /(1-z)$ and $L \ln ^{2}(1-z) /(1-z)$. Note that the convolution as well as exponentiation can not give the true complete sub-leading formula. And, contrary to the case of pure photonic radiation, they are unable even to cover the full leading log result: the term with $P^{(1)}$ is not reproduced (this term however has a small coefficient and no any $\ln (1-z)$, so it is negligible numerically in our task). This term corresponds to the physical situation (Feynman diagrams), when the extra photon is attached to the secondary pair.

In fact the convolution gives a part of sub-leading terms coming from the kinematical regions, where both the pair and the photon are either soft or emitted collinearly to the parent particle momentum, while there are other sources for the corrections, like, for instance, emission of a collinear pair and a large-angle photon. But we believe, that the main terms with reinforcements are reproduced correctly: that follows from the general experience in leading $\log$ calculations. Moreover, for the case of pure photonic radiation this has been checked by direct perturbative calculations.

In this way we received the expressions for the third order pair radiator. For leptonic non-singlet pairs we have

$$
\begin{aligned}
H_{\Theta}^{(3) f}(z) & =\left(\frac{\alpha}{\pi}\right)^{3} L_{e}\left[\frac{1+z^{2}}{1-z} L_{f}^{2}\left(\frac{2}{3} \ln (1-z)-\frac{1}{3} \ln z+\frac{1}{2}\right)+L_{f}^{2}\left(\frac{1+z}{6} \ln z-\frac{1-z}{3}\right)\right. \\
& +\frac{1+z^{2}}{1-z} L_{f}\left(2 \ln ^{2}(1-z)-\frac{11}{9} \ln (1-z)-\frac{9}{4}-\frac{4}{3} \zeta(2)-2 \ln z \ln (1-z)\right. \\
& \left.+\frac{1}{3} \ln ^{2} z+\frac{11}{18} \ln z\right)+L_{f}\left(-\frac{8}{3}(1-z) \ln (1-z)+\frac{2}{3}(1+z) \ln z \ln (1-z)\right. \\
& \left.-\frac{1}{6}(1+z) \ln ^{2} z+\frac{4}{9}(1-5 z) \ln z+\frac{2}{3}(1+z) \operatorname{Li}_{2}(1-z)+\frac{19}{9}(1-z)\right) \\
& +\frac{1+z^{2}}{1-z}\left(\frac{16}{9} \ln ^{3}(1-z)-\frac{7}{3} \ln ^{2}(1-z)+\frac{67}{27} \ln (1-z)-\frac{16}{3} \zeta(2) \ln (1-z)\right. \\
& -\frac{8}{3} \ln ^{2} \ln ^{2}(1-z)+\frac{7}{3} \ln z \ln (1-z)+\frac{5}{6} \ln z \ln (1-z)-\frac{1}{3} \operatorname{Li}_{2}(1-z) \ln (1-z) \\
& -\frac{1}{18} \ln ^{3} z-\frac{31}{72} \ln ^{2} z-\frac{67}{54} \ln z-\frac{2}{3} \operatorname{Li}_{2}(1-z) \ln z+\frac{8}{3} \zeta(2) \ln z-\frac{1}{4} \operatorname{Li}_{2}(1-z) \\
& \left.\left.-\frac{5}{3} \mathrm{~S}_{1,2}(1-z)-\frac{4}{3} \zeta(2)+4 \zeta(3)+\frac{1073}{162}\right)+\mathcal{O}(1)\right],
\end{aligned}
$$




$$
\mathrm{Li}_{2}(x)=-\int_{0}^{x} \frac{\mathrm{d} y}{y} \ln (1-y), \quad \mathrm{S}_{1,2}(x)=\frac{1}{2} \int_{0}^{x} \frac{\mathrm{d} y}{y} \ln ^{2}(1-y) .
$$

The large logarithm $L_{f}=\ln \left(s / m_{f}^{2}\right)$ stands for different lepton flavours: $f=e, \mu, \tau$. The corresponding soft+virtual contribution is given by

$$
\begin{aligned}
H_{\Delta}^{(3) f} & =\left(\frac{\alpha}{\pi}\right)^{3} L_{e}\left[\frac{8}{9} \ln ^{4} \Delta+\ln ^{3} \Delta\left(\frac{4}{3} L_{f}-\frac{14}{9}\right)+\ln ^{2} \Delta\left(\frac{2}{3} L_{f}^{2}-\frac{11}{9} L_{f}+\frac{67}{27}-\frac{16}{3} \zeta(2)\right)\right. \\
& +\ln \Delta\left(L_{f}^{2}-\frac{9}{2} L_{f}-\frac{8}{3} \zeta(2) L_{f}+8 \zeta(3)-\frac{8}{3} \zeta(2)+\frac{1073}{81}\right)+L_{f}^{2}\left(\frac{3}{8}-\frac{2}{3} \zeta(2)\right) \\
& \left.+L_{f}\left(-\frac{17}{8}+\frac{8}{3} \zeta(3)+\frac{20}{9} \zeta(2)\right)+\frac{1543}{216}-\frac{22}{9} \zeta(3)-\frac{413}{54} \zeta(2)+\frac{8}{15} \zeta^{2}(2)\right] .
\end{aligned}
$$

The cancellation of the auxiliary parameter $\Delta$ was checked analytically and achieved numerically. The largest contribution appears at the $Z$-peak, where the integral over $z$ is practically limited by the $Z$-boson width: $\left(z_{\min }, 1\right) \rightarrow\left(1-\Gamma_{Z} / M_{Z}, 1\right)$. And therefore integrals from terms, like $\ln (1-z) /(1-z)$, are large. We checked that for the real hard emission there is an agreement between the most important terms in $H_{\Theta}^{(3)}(z)$ and the corresponding terms in expansion of the exponentiated formula from Ref. 9]. Such a correspondence between the exponentiation and convolution procedures is well known also in the case of pure photonic radiation.

A similar expression was derived for the case of hadronic pairs:

$$
\begin{aligned}
& H_{\Theta}^{(3) \mathrm{h}}(z)=\left(\frac{\alpha}{\pi}\right)^{3} L_{e}\left\{\frac{1+z^{2}}{1-z} L_{h}^{2} R(\infty)\left(\frac{2}{3} \ln (1-z)-\frac{1}{3} \ln z+\frac{1}{2}\right)\right. \\
& +L_{h}^{2} R(\infty)\left(\frac{1+z}{6} \ln z-\frac{1-z}{3}\right)+\frac{1+z^{2}}{1-z} L_{h}\left[R ( \infty ) \left(2 \ln ^{2}(1-z)+\ln (1-z)\right.\right. \\
& -\frac{8}{3} \ln 2 \ln (1-z)-2 \ln (z) \ln (1-z)+\frac{1}{3} \ln ^{2} z-\frac{1}{2} \ln z+\frac{4}{3} \ln 2 \ln z-\frac{7}{12}-2 \ln 2 \\
& \left.\left.-\frac{4}{3} \zeta(2)\right)+R_{0}\left(\frac{4}{3} \ln (1-z)+1-\frac{2}{3} \ln z\right)\right]+L_{h}\left[R ( \infty ) \left(-\frac{8}{3}(1-z) \ln (1-z)\right.\right. \\
& +\frac{2}{3}(1+z) \ln (z) \ln (1-z)-\frac{1}{6}(1+z) \ln ^{2}(z)+\frac{2}{3}(1+z) \operatorname{Li}_{2}(1-z)+\ln z \\
& \left.-\frac{5}{3} z \ln z+\frac{2}{3} \ln 2(1+z) \ln z+\frac{4}{3}(1-z) \ln 2+1-z\right) \\
& \left.+\quad R_{0}\left(\frac{1}{3}(1+z) \ln z-\frac{2}{3}(1-z)\right)\right]+\frac{1+z^{2}}{1-z}\left[R ( \infty ) \left(\frac{16}{9} \ln ^{3}(1-z)+\ln ^{2}(1-z)\right.\right. \\
& -4 \ln 2 \ln ^{2}(1-z)-4 \zeta(2) \ln (1-z)-2 \ln 2 \ln (1-z)+\frac{8}{3} \ln ^{2} 2 \ln (1-z) \\
& -\frac{1}{3} \operatorname{Li}_{2}(1-z) \ln (1-z)+\frac{5}{6} \ln ^{2} z \ln (1-z)-\frac{8}{3} \ln z \ln ^{2}(1-z) \\
& -\ln z \ln (1-z)+4 \ln 2 \ln z \ln (1-z)-\frac{5}{3} \mathrm{~S}_{1,2}(1-z)-\frac{2}{3} \operatorname{Li}_{2}(1-z) \ln z \\
& -\frac{1}{4} \operatorname{Li}_{2}(1-z)-\frac{1}{18} \ln ^{3} z+\ln 2 \ln z+2 \zeta(2) \ln z+\frac{1}{8} \ln ^{2} z-\frac{2}{3} \ln 2 \ln ^{2} z \\
& \left.-\frac{4}{3} \ln ^{2} 2 \ln z+\frac{8}{3} \ln 2 \zeta(2)+\frac{7}{6} \ln 2+2 \ln ^{2} 2-\zeta(2)+\frac{10}{3} \zeta(3)+\frac{5}{8}\right)
\end{aligned}
$$




$$
\begin{aligned}
& +R_{0}\left(2 \ln ^{2}(1-z)-\frac{8}{3} \ln 2 \ln (1-z)+\ln (1-z)-2 \ln z \ln (1-z)\right. \\
& \left.-\frac{1}{2} \ln z+\frac{1}{3} \ln ^{2} z+\frac{4}{3} \ln 2 \ln z-\frac{7}{12}-2 \ln 2-\frac{4}{3} \zeta(2)\right) \\
& \left.\left.+R_{1}\left(\frac{4}{3} \ln (1-z)+1-\frac{2}{3} \ln z\right)\right]+\mathcal{O}(1)\right\} \\
& H_{\Delta}^{(3) \mathrm{h}}=\left(\frac{\alpha}{\pi}\right)^{3} L_{e}\left\{\ln ^{4} \Delta \frac{8}{9} R(\infty)+\ln ^{3} \Delta\left[R(\infty)\left(\frac{4}{3} L_{h}+\frac{2}{3}-\frac{8}{3} \ln 2\right)+\frac{4}{3} R_{0}\right]\right. \\
& +\ln ^{2} \Delta\left[R(\infty)\left(\frac{2}{3} L_{h}^{2}+L_{h}-\frac{8}{3} \ln 2 L_{h}-2 \ln 2+\frac{8}{3} \ln ^{2} 2-4 \zeta(2)\right)\right. \\
& \left.+R_{0}\left(\frac{4}{3} L_{h}+1-\frac{8}{3} \ln 2\right)+\frac{4}{3} R_{1}\right]+\ln \Delta\left[R ( \infty ) \left(L_{h}^{2}-\frac{8}{3} \zeta(2) L_{h}\right.\right. \\
& -\quad \frac{7}{6} L_{h}-4 \ln 2 L_{h}+\frac{16}{3} \ln 2 \zeta(2)+\frac{7}{3} \ln 2+4 \ln ^{2} 2-2 \zeta(2)+\frac{20}{3} \zeta(3) \\
& \left.\left.+\frac{5}{4}\right)+R_{0}\left(2 L_{h}-\frac{8}{3} \zeta(2)-\frac{7}{6}-4 \ln 2\right)+2 R_{1}\right] \\
& +\left(\frac{3}{8}-\frac{2}{3} \zeta(2)\right) L_{h}^{2} R(\infty)+L_{h} R(\infty)\left(\frac{8}{3} \zeta(3)+\frac{8}{3} \ln 2 \zeta(2)-\frac{7}{8}-\frac{3}{2} \ln 2\right) \\
& +\quad L_{h} R_{0}\left(\frac{3}{4}-\frac{4}{3} \zeta(2)\right)+R(\infty)\left(\frac{3}{2} \ln ^{2} 2+\frac{7}{4} \ln 2+\zeta(3)-\frac{16}{3} \zeta(3) \ln 2-\frac{4}{5} \zeta^{2}(2)\right. \\
& \left.-\frac{5}{12} \zeta(2)-\frac{8}{3} \zeta(2) \ln ^{2} 2+\frac{23}{48}\right)+R_{0}\left(\frac{8}{3} \zeta(3)+\frac{8}{3} \zeta(2) \ln 2-\frac{3}{2} \ln 2-\frac{7}{8}\right) \\
& \left.+R_{1}\left(\frac{3}{4}-\frac{4}{3} \zeta(2)\right)\right\}, \quad L_{h}=\ln \frac{s}{m_{\pi}^{2}} \text {. }
\end{aligned}
$$

Quantities $R(\infty), R_{0,1}$ are defined in Ref. [4]. In contrast to Ref. [9] we extended the hadronic pair contribution to the third order dynamically, but not by a static coefficient. That is really important at LEP2 energies.

The LLA leptonic contributions, which were not reproduced by the convolution (the terms with $P^{(1)}$ and $P^{(1)} \otimes R^{s}$ functions in Eq. (5)), should be added to Eqs. (10), (11). The third order effect appeared to be not small numerically. So, we have to look also at the fourth order. The corresponding contribution is estimated by means of the leading logs [1] (non-singlet channel only):

$$
\mathrm{d} \sigma_{e}^{(4)}=\int \mathrm{d} z \tilde{\sigma}(z s)\left(\frac{\alpha}{2 \pi}\left(L_{e}-1\right)\right)^{4}\left[\frac{1}{12} P^{(3)}(z)+\frac{11}{216} P^{(2)}(z)+\frac{1}{108} P^{(1)}(z)\right] .
$$

Only the term with the $P^{(3)}$ splitting function [10] seems to be numerically significant. It agrees with the corresponding term in the expansion of exponentiated formula in Ref. [9]. The size of the fourth order, which is estimated from the above formula for electron pairs, is rather small. So, we can be less rigorous: in order to estimate the fourth order contribution from other kinds of pairs and the large sub-leading terms, we drop them simultaneously. That was checked in lower orders to be a reasonable approximation. So, in the $\mathcal{O}\left(\alpha^{4}\right)$ we keep only the leading logarithmic formula (14) for non-singlet electron pairs.

In Table 2 we present the results for the different contributions of pair corrections to the process of annihilation into hadrons. The value of correction due to pairs is defined in respect 


\begin{tabular}{|l|c|c|c|c|c|c|c|}
\hline & $e(\mathrm{NS})$ & $e(\mathrm{~S})$ & $e(\mathrm{NS} \times \mathrm{S})$ & $\mu$ & $\tau$ & hadr. & sum(NS) \\
\hline & \multicolumn{7}{|c|}{$E_{\mathrm{cm}}=88.1867 \mathrm{GeV}$} \\
\hline $\mathcal{O}\left(\alpha^{2}\right)$ & -2.11 & +1.13 & -0.02 & -0.34 & -0.04 & -0.70 & -3.19 \\
$\mathcal{O}\left(\alpha^{3}\right)$ & -1.51 & +1.12 & -0.02 & -0.26 & -0.04 & -0.35 & -2.16 \\
$\mathcal{O}\left(\alpha^{4}\right)$ & -1.70 & +1.12 & -0.02 & -0.26 & -0.04 & -0.35 & -2.36 \\
\hline & \multicolumn{7}{|c|}{$E_{\mathrm{cm}}=91.1867 \mathrm{GeV}$} \\
\hline $\mathcal{O}\left(\alpha^{2}\right)$ & -2.25 & +0.16 & -0.01 & -0.38 & -0.03 & -0.77 & -3.43 \\
$\mathcal{O}\left(\alpha^{3}\right)$ & -1.69 & +0.15 & -0.01 & -0.30 & -0.04 & -0.42 & -2.44 \\
$\mathcal{O}\left(\alpha^{4}\right)$ & -1.78 & +0.15 & -0.01 & -0.30 & -0.04 & -0.42 & -2.54 \\
\hline & \multicolumn{7}{|c|}{$E_{\mathrm{cm}}=94.1867 \mathrm{GeV}$} \\
\hline $\mathcal{O}\left(\alpha^{2}\right)$ & +1.25 & +0.55 & -0.05 & +0.09 & -0.02 & +0.05 & +1.37 \\
$\mathcal{O}\left(\alpha^{3}\right)$ & +0.13 & +0.54 & -0.05 & -0.10 & -0.03 & -0.21 & -0.22 \\
$\mathcal{O}\left(\alpha^{4}\right)$ & +0.43 & +0.54 & -0.05 & -0.10 & -0.03 & -0.21 & +0.08 \\
\hline & \multicolumn{7}{|c|}{$E_{\mathrm{cm}}=189 \mathrm{GeV}$} \\
\hline $\mathcal{O}\left(\alpha^{2}\right)$ & +6.33 & +31.80 & -1.32 & +1.93 & +0.63 & +5.30 & +14.20 \\
$\mathcal{O}\left(\alpha^{3}\right)$ & +7.13 & +30.48 & -1.32 & +2.11 & +0.68 & +5.83 & +15.76 \\
$\mathcal{O}\left(\alpha^{4}\right)$ & +7.08 & +30.48 & -1.32 & +2.11 & +0.68 & +5.83 & +15.71 \\
\hline & \multicolumn{7}{|c|}{$E_{\mathrm{cm}}=206 \mathrm{GeV}$} \\
\hline $\mathcal{O}\left(\alpha^{2}\right)$ & +6.43 & +40.38 & -1.41 & +2.00 & +0.68 & +5.56 & +14.68 \\
$\mathcal{O}\left(\alpha^{3}\right)$ & +7.32 & +38.74 & -1.40 & +2.22 & +0.74 & +6.19 & +16.48 \\
$\mathcal{O}\left(\alpha^{4}\right)$ & +7.28 & +38.74 & -1.40 & +2.22 & +0.74 & +6.19 & +16.44 \\
\hline
\end{tabular}

Table 2: Different contributions to $\delta_{\mathrm{ISP}}^{e^{+} e^{-} \rightarrow \text { hadr. }}$ in permil.

to the cross section for annihilation into hadrons with pure photonic corrections taken into account:

$$
\delta_{\mathrm{ISP}}=10^{3}\left(\frac{\sigma_{\mathrm{ISP}, \gamma}^{\mathrm{RC}}}{\sigma_{\gamma}^{\mathrm{RC}}}-1\right) .
$$

The cut-off on the primary pair mass $s^{\prime}>z_{\min } \cdot s, \quad z_{\min }=0.01$, acts in the same way on real photons and secondary pairs.

In Table 3 we present the values of $\delta_{\mathrm{ISP}}^{e^{+} e^{-} \rightarrow \mu^{+} \mu^{-}}$for different cuts and beam energies. In the first two columns we give the contributions of singlet pairs and their interference with the non-singlet ones. The latter, in principle, should be counted as a part of the signal. The third column contains the NS contribution (our best estimate with all pair flavours summed up). It can be compared with our implementation of exponentiated pair corrections. Note, that in both the last two columns the contribution of hadronic pairs is not exponentiated, but it is supplied according to our approach (we used result [4 supplied with our third order contribution (12), (13)).

For the column "[7]", the known complete second order result was supplied by the NS higher order part of the exponentiated formula, derived in this paper. The discrepancy with our best estimate originates from an improper reproduction of the third order correction by the exponentiation in Ref. [7]. Note that the largest difference appears at LEP2 energies for small $z_{\min }$ values, which means the dominance of the effect of radiative return to the $Z$ peak due to hard pair emission. While the exponentiation [7] is well justified only for soft 
radiation, which was the only one relevant at LEP1. The last column was calculated by using the exponentiated formula of Ref. 9]. The approximation for the higher order hadronic contribution (static coefficient before the second order contribution), suggested in that paper has sense only at LEP1 energy on the top of the peak and the left wing; everywhere else it fails. Errors in the third column represent our estimate of theoretical uncertainties (see the Conclusions).

As could be seen from Table 3, the contribution of singlet pair production becomes significant for small values of $z_{\text {min }}$. In data analysis such events are supposed 2 to be extracted from the data together with the two-photon (multi-peripheral) process [12. We underline, that the procedure should be accurate and well understood, because the events with singlet pairs and multi-peripheral production have completely different kinematics and signatures in the detector. At LEP2 energies the contribution of singlet pairs becomes really important, if the returning to the $Z$-peak is allowed (for $z_{\text {min }} \lesssim 0.25$ ). The large contribution is coming from the peculiar $1 / z$ term in the function $R^{s}$, Eq. (6) . Note, that this function is multiplied by the kernel cross section which contains $z^{-1}$ too. In this way the second order correction due to collinear emission of singlet pairs can give several percent. This did not happen at LEP1, because the kernel cross section outside the $Z$-peak is relatively small.

The difference between the column "non-singlet" and the last one "[9]" is coming just from the difference of the perturbative and exponentiated treatment of higher order leptonic pair corrections. The difference is less on the right wing of the $Z$-peak, where the real radiation is dominant, because it provides returning to the peak. For real radiation we expanded the exponentiated formula and saw an agreement in the main terms. On the other side of the peak (and on the top) the virtual and soft corrections are dominant, because any real hard radiation leads to a huge reduction of the kernel cross section in Eq. (4). In this case we have to compare the soft+virtual part of our radiator $H_{\Delta}$ with the integral

$$
\int_{1-\Delta}^{1} \mathrm{~d} z\left(\rho(z)-\rho^{\gamma}(z)\right),
$$

where $\rho(z)$ is the exponentiated formula [9], and $\rho^{\gamma}(z)$ is the pure photonic exponentiated radiator 13 . The latter is in perfect agreement with the perturbative results both in the hard and soft+virtual regions, while the formula with simultaneous exponentiations of photonic and pair radiation has a very peculiar structure for $z \rightarrow 1$, and so Eq. (16) can be hardly expanded in a series in $\alpha$ to compare with the perturbative results. The problem is seen in the region of very soft photons $(z \rightarrow 1)$ below the threshold of pair production, where the part of the exponentiated formula, which describes pairs, still represents a sharp dependence on $z$-value, instead of giving a constant coefficient from virtual pairs.

It is worth to note the weak dependence on the $z_{\text {min }}$ parameter of the corrections at LEP1 energies. So, the bulk of the corrections is given by soft and virtual pairs, which are not mixed during the observation with the primary pair from electron positron annihilation.

\section{Conclusions}

First we have to remind about the effect of the initial-final interference (IFI) in pair corrections. We suggest to estimate it by using the known effect for the IFI in photonic corrections. The 


\begin{tabular}{|c|c|c|c|c|c|}
\hline$E_{\mathrm{cm}}[\mathrm{GeV}]$ & singlet & S $\times$ NS & non-singlet & {$[7]$} & ]$\left.^{*}\right]^{* 1}$ \\
\hline \multicolumn{7}{|c|}{$z_{\min }=0.01$} \\
\hline 88.1867 & +5.61 & -0.06 & $-1.96 \pm 0.28$ & -1.52 & -1.76 \\
91.1867 & +0.81 & -0.01 & $-2.48 \pm 0.22$ & -2.16 & -2.31 \\
94.1867 & +2.78 & -0.07 & $+0.22 \pm 0.41$ & +0.31 & +0.16 \\
189 & +51.03 & -1.08 & $+11.78 \pm 0.82$ & +14.17 & +11.39 \\
206 & +56.69 & -1.13 & $+12.17 \pm 0.88$ & +14.77 & +11.74 \\
\hline \multicolumn{7}{|c|}{$z_{\min }=0.1$} \\
\hline 88.1867 & +0.24 & -0.03 & $-2.17 \pm 0.28$ & -1.78 & -1.94 \\
91.1867 & +0.03 & -0.01 & $-2.51 \pm 0.22$ & -2.20 & -2.33 \\
94.1867 & +0.12 & -0.05 & $+0.12 \pm 0.41$ & +0.19 & +0.07 \\
189 & +12.48 & -0.91 & $+10.80 \pm 0.81$ & +12.81 & +10.53 \\
206 & +18.14 & -0.95 & $+11.13 \pm 0.86$ & +13.40 & +10.83 \\
\hline \multicolumn{7}{|c|}{$z_{\min }=0.5$} \\
\hline 88.1867 & +0.00 & -0.01 & $-2.41 \pm 0.28$ & -2.04 & -2.17 \\
91.1867 & +0.00 & -0.01 & $-2.54 \pm 0.22$ & -2.23 & -2.36 \\
94.1867 & +0.00 & -0.04 & $-0.00 \pm 0.41$ & +0.06 & -0.05 \\
189 & +0.02 & -0.05 & $-1.05 \pm 0.09$ & -0.80 & -0.96 \\
206 & +0.02 & -0.05 & $-1.09 \pm 0.08$ & -0.83 & -1.00 \\
\hline \multicolumn{7}{|c|}{$z_{\min }=0.9$} \\
\hline 88.1867 & +0.00 & -0.01 & $-2.58 \pm 0.29$ & -2.20 & -2.33 \\
91.1867 & +0.00 & -0.01 & $-2.59 \pm 0.22$ & -2.28 & -2.41 \\
94.1867 & +0.00 & -0.04 & $-0.32 \pm 0.41$ & -0.25 & -0.37 \\
189 & +0.00 & -0.02 & $-2.33 \pm 0.15$ & -2.03 & -2.22 \\
206 & +0.00 & -0.02 & $-2.40 \pm 0.16$ & -2.10 & -2.29 \\
\hline
\end{tabular}

Table 3: Radiative correction $\delta$ for different cuts.

bulk of the IFI pair correction is provided by the terms of the order $\mathcal{O}\left(\alpha^{2} L\right)$ (the sub-leading logarithms). The terms are coming from the kinematical region of small-invariant-mass real pairs (and from the corresponding region of integration over virtual pairs). Thus, one can use the factorization of the correction factors, corresponding to the photon emission and the pair production. So, we just multiply the IFI photonic correction by the factor $\alpha L /(3 \pi)$ and receive the estimate for the IFI pair $\mathrm{RC}\left(L \equiv \ln \left(s / m_{e, \mu, \pi}^{2}\right)\right)$. The typical value of the photonic IFI RC at LEP energies is a few permil, therefore one can safely neglect the corresponding pair contribution. Note, that in this way we considered only the non-singlet mechanism of pair production. The interference of the initial state singlet pair production amplitudes with the final state NS ones is expected to be of the same magnitude.

Let us discuss now the uncertainty in the pair corrections. We mean just the indefiniteness in the treatment of hadronic pairs and of the $\mathcal{O}\left(\alpha^{3}\right)$ (and higher order) leptonic pair corrections. Other sources, relevant for concrete experiments, like the problem to distinguish between the primary and secondary pair or the proper subtraction of the background, have to be discussed elsewhere [2].

First we suppose, that $15 \%$ is a reasonably safe estimate for the error in the second order hadronic pair contribution itself. In this case we use a phenomenological approach based on the measurements of electron-positron annihilation into hadrons at low energies. The 
absence on the market of a plug-and-play parameterization of the $R$-function (ratio of the annihilation cross sections into hadrons and muons) does not allow to put a better precision level. For our computations of the FS hadronic pairs a simple parameterization of the function was constructed. The dispersion relations were used to look at the corresponding vacuum polarization effect and check the function. The stability in respect of the fluctuations in involved data was verified. A good agreement was observed with the published numbers on hadronic pair corrections [4, 5] in both the initial and the final states.

The uncertainty of the higher order leptonic pairs is derived as follows. We assume that the leading $\operatorname{logs} L$, especially the ones multiplied by a power of $\ln \left(M_{Z}^{2} / \Gamma_{Z}^{2}\right)$, are taken into account completely. As stated above, all sub-leading terms can not be generated neither by convolution nor by exponentiation. Therefore, we can estimate the error of the third order contribution to be about $1 / L$ (actually we take $1 / 5$ ) of its own size. For the third order hadronic contribution, derived by means of convolution, we should take into account also the intrinsic uncertainty in hadronic pairs, discussed above. As concerning the fourth and higher orders, we suggest to take the error just of the order $\mathcal{O}\left(\alpha^{4} L^{4}\right) e^{+} e^{-}$contribution (for all flavours together).

The situation is better for the case of the final state pair corrections: the phenomenological treatment of hadronic pairs and the omitted effect of the interplay between the FS photons and pairs can not lead to more than 10 percent error in the FS pair RC, which is already small itself. Remind also the interference between the initial and final pair production amplitudes, discussed above. Collecting the sources of theoretical uncertainties, involved into our approach, we conclude: i) at LEP1 energies the error is about 0.4 permil on the wings and 0.2 permil on the top of the resonance; ii) at LEP2, when the radiative return to the $Z$-peak is forbidden, we have a reasonably good error of about 0.1 permil (for the realistic cut $z_{\min }=0.7225$ ); iii) when the radiative return is allowed, the size of the error can reach 1 permil.

The approach to pair corrections described here is implemented into the semi-analytical

code ZFITTER v.6.30 [6]. A treatment of higher order pairs (with pair-photon interplay) is available also in TOPAZO [14, GEANTLE [15], KORALW [16], and some other codes. Some comparisons of the approaches were done in Ref. [2].

\section{Acknowledgments}

I am grateful to D. Bardin and G. Passarino for valuable discussions.

\section{References}

[1] D.Y. Bardin, M. Grunewald and G. Passarino, hep-ph/9902452.

[2] M. Kobel et al. [Two Fermion Working Group], hep-ph/0007180.

[3] F.A. Berends, W.L. van Neerven and G.J.H. Burgers, Nucl. Phys. B 297 (1988) 429 [Erratum-ibid. B 304 (1988) 921].

[4] B.A. Kniehl, M. Krawczyk, J.H. Kuhn and R.G. Stuart, Phys. Lett. B 209 (1988) 337.

[5] A.H. Hoang, J.H. Kuhn and T. Teubner, Nucl. Phys. B 455 (1995) 3 hep-ph/9507255. 
[6] D.Y. Bardin, P. Christova, M. Jack, L. Kalinovskaya, A. Olchevski, S. Riemann and T. Riemann, Comput. Phys. Commun. 133 (2001) 229 hep-ph/9908433.

[7] E.A. Kuraev and V.S. Fadin, Sov. J. Nucl. Phys. 41 (1985) 466.

[8] S. Catani and L. Trentadue, JETP Lett. 51 (1990) 83.

[9] S. Jadach, M. Skrzypek and M. Martinez, Phys. Lett. B 280 (1992) 129.

[10] M. Skrzypek, Acta Phys. Polon. B23 (1992) 135.

[11] A.B. Arbuzov, Phys. Lett. B470 (1999) 252 hep-ph/9908361.

[12] D. Decamp et al. [ALEPH Collaboration], Z. Phys. C 48 (1990) 365.

[13] S. Jadach, M. Skrzypek and B. F. L. Ward, Phys. Lett. B 257 (1991) 173.

[14] G. Montagna, O. Nicrosini, F. Piccinini and G. Passarino, Comput. Phys. Commun. 117, 278 (1999) hep-ph/9804211.

[15] D. Bardin, J. Biebel, D. Lehner, A. Leike, A. Olchevski and T. Riemann, Comput. Phys. Commun. 104 (1997) 161 hep-ph/9612409.

[16] S. Jadach, W. Placzek, M. Skrzypek, B. F. Ward and Z. Was, Comput. Phys. Commun. 119 (1999) 272 hep-ph/9906277. 